\title{
Fusion of Multi-band SAR Images Based on Tetrolet Transform
}

\author{
Zihong Chen ${ }^{1, a}$, BaoHong Yuan ${ }^{2, b}$, Dexiang Zhang ${ }^{1,2, c}$, Jingjing Zhang ${ }^{1, d}$ \\ ${ }^{1}$ The School of Electrical Engineering and Automation, Anhui University, Hefei 230601, China \\ ${ }^{2}$ The School of Electronic and Electrical Engineering of Anhui Sanlian University, Hefei, 230601, \\ China \\ a chenzihong315@126.com, ${ }^{b}$ baohong_yuan@163.com \\ ${ }^{c}$ zdxdzxy@126.com (corresponding author), ${ }^{\mathrm{d}}$ helenzjj@aiofm.ac.cn
}

Keywords: SAR image, Tetrolet transform, image fusion, variance

\begin{abstract}
An efficient SAR image fusion algorithm for multi-band SAR images based on tetrolet transform is proposed. Tetrolets are Haar-type wavelets whose supports are tetrominoes which are shapes made by connecting four equal-sized squares. Firstly, the multi-band SAR images can be decomposed into low-frequency coefficients and high-frequency coefficients with multi-scales and multi-directions using tetrolet transform. For the low-frequency coefficients, the average fusion method is used. For the each directional high frequency sub-band coefficients, the larger value of variance information measurement is used to select the better coefficients for fusion. At last the fused image can be obtained by utilizing inverse transform for fused tetrolet coefficients. Experimental results show that compared with traditional algorithm, the proposed algorithm can get better visual effect and the significant information of original image like textures and contour details is well maintained.
\end{abstract}

\section{Introduction}

Synthetic aperture radar (SAR) remote sensing images can get multi-resolution and multi-frequency band image data. But the different kind of SAR image sensor can only capture different image information. Data fusion is capable of integrating different imagery data creating more information than can be derived from a single sensor. Image fusion is a very effective technique which can be applied to many remote sensing areas such as classification, monitoring of environmental surveillance and man-made target tracking ${ }^{[1]}$.

The current definition of sensor fusion is very broad and the fusion can take place in three different processing levels according to the stage at which the fusion takes place: pixel level, feature level and decision level. Geometrical features information in images, like edges and contours, play the most important roles in many image processing areas, such as compression, denoising and image fusion. Many researchers recognized that multi-scale transforms are very useful for analyzing the information of images processing ${ }^{[2]}$.

In order to design anisotropic basis functions that can capture anisotropic sparse representation of geometrical information in images. Many approaches based on multi-scale transforms, such as gradient pyramid, wavelet transform and contourlet transform, have been proposed in recent years ${ }^{[3]}$. Many new anisotropic image decomposition methods have already been considered and exploited by adaptive or non-adaptive processing, such as Bandetlets, Curvelets and Contourlets ${ }^{[4]}$.The separable wavelet transform has been widely applied in the field of image processing and provided good processing affect. However, the wavelet transform is limited by the spatial isotropy of the wavelet basis functions as well as the lack of directionality.

This paper introduces a new image fusion method based on tetrolet transform. The proposed method solves the problem of losing edge information for wavelet based fusion method. The proposed method is especially designed for sparse image approximation due to the non-redundance of the basis functions ${ }^{[5]}$. 


\section{Tetrolet transform}

Tetrolets are Haar-type wavelets whose supports are tetrominoes which are shapes made by connecting four equal-sized squares. The corresponding fast filter bank algorithm is simple but very effective. In every level of the filter bank algorithm we divide the low-pass image into $4 \times 4$ blocks. Then in each block we determine a local tetrolet basis which is adapted to the image geometry in this block. The idea of tetrolet transform is to allow more general partitions which capture the image local geometry by bringing the "tiling by tetrominoes" problem into play ${ }^{[7]}$.

Tetrominoes were introduced by Golomb in [6]. We can obtain a tetromino by connecting four equal sized squares, each connected by edges, not merely at their corners. Disregarding rotations and reflections there are five different shapes, as shown in figure 1:
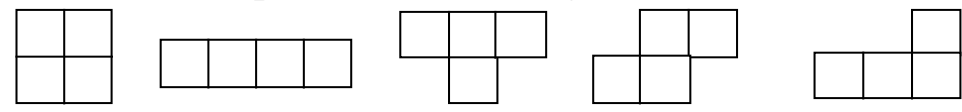

Fig. 1 The five free tetrominoes

There are 117 solutions for disjoint covering of a $4 \times 4$ board with four tetrominoes. We have 22 fundamental configurations (disregarding rotations and reflections).

We start with the input image $a^{0}=(a[i, j])_{i, j=0}^{N-1}$ with $N=2^{J}, J \in N$. In the $\mathrm{r}$ th-level, $r=1,2, \ldots, J-1$, we apply the following computations.

1. Divide the low-pass image $\mathrm{a}^{r-1}$ into blocks $\mathrm{Q}_{i, j}$ of $i, j=0,1, \ldots, N / 2^{r+1}-1$.

2. Find in each block the sparsest tetrolet representation. In each block $\mathrm{Q}_{i, j}$ we compute the pixel averages for every admissible tetromino covering $c=1,2, \ldots, 117$, we can determine the lowpass part

$$
\mathrm{a}^{\mathrm{r},(\mathrm{c})}[s]=\sum_{(m, n) \in I_{s}^{(c)}} \varepsilon[0, L(m, n)] a^{r-1}[m, n]
$$

as well as the three high-pass parts for $l=1,2,3$

$$
w_{l}^{r,(c)}=\left(\sum_{(m, n) \in I_{s}^{(c)}} \varepsilon[l, L(m, n)] a^{r-1}[m, n]\right)_{s=0}^{3}
$$

where the coefficients $\varepsilon[l, m], l, m=0,1,2,3$ are entries from the Haar wavelet transform matrix

$$
W=(\varepsilon[l, m])_{l, m=0}^{3}=\frac{1}{2}\left[\begin{array}{cccc}
1 & 1 & 1 & 1 \\
1 & 1 & -1 & -1 \\
1 & -1 & 1 & -1 \\
1 & -1 & -1 & 1
\end{array}\right]
$$

Then we choose the covering $c^{*}$ such that the $l_{1}$-norm of the tetrolet coefficients becomes minimal

$$
c^{*}=\arg \min _{c} \sum_{l=1}^{3} \sum_{s=0}^{3}\left|w_{l}^{r,(c)}[s]\right|
$$

Hence, for every block $Q_{i, j}$ we get optimal tetrolet decomposition $\left[a^{r,\left(c^{*}\right)}, w_{1}^{r,\left(c^{*}\right)}, w_{2}^{r,\left(c^{*}\right)}, w_{3}^{r,\left(c^{*}\right)}\right]$.

3. In order to be able to apply further levels of the tetrolet decomposition algorithm, rearrange the low-pass and high-pass coefficients of each block into a $2 \times 2$ block.

$$
a_{\mid Q_{i, j}}^{r}=R\left(a^{r,\left(c^{*}\right)}\right)=\left[\begin{array}{ll}
a^{r,\left(c^{*}\right)}[0] & a^{r,\left(c^{*}\right)}[2] \\
a^{r,\left(c^{*}\right)}[1] & a^{r,\left(c^{*}\right)}[3]
\end{array}\right]
$$

and in the same way $w_{l \mid Q_{i, j}}^{r}=R\left(w_{l}^{r,\left(c^{*}\right)}\right)$ 
4. After finding a sparse representation in every block $\mathrm{Q}_{i, j}$, we store the low-pass matrix $\mathrm{a}^{r}$ and the high-pass matrices $w_{l}^{r}$, replacing the low-pass image $a^{r-1}$ by the matrix

$$
a^{r-1}=\left[\begin{array}{ll}
a^{r} & w_{2}^{r} \\
w_{1}^{r} & w_{3}^{r}
\end{array}\right]
$$

5. Apply step 1 to 4 to the low-pass image we can get multi-scale and multi-direction tetrolet decompose.

\section{Tetrolet Based Image Fusion}

The adaptivity of the tetrolet transform to the local geometric structures comes from the optimization process used in the filter bank algorithm. We apply the Tetrolet transform to image fusion so as to create new fused images that have more information than the source images, and are more suited to the purposes of human visual perception, object detection and target recognition.

The each original image is decomposed in multi-scale and multi-direction at different level by tetrolet transform, so that an original image is decomposed into several subbands. The low frequency sub-band image coefficients shows the image approximate characteristic. For the low frequency coefficients fusing, the fused coefficients are obtained with average rule. The fusion rule can describe as:

$$
T_{F}(m, n)=\left(T_{A}(m, n)+T_{B}(m, n)\right) / 2
$$

where $T_{A}(m, n)$ and $T_{B}(m, n)$ is low coefficients of image $A$ and image $B$ respectively. $T_{F}(m, n)$ is low coefficients of fused image.

In tetrolet transform, divide the low-pass image into blocks of size $4 \times 4$, the 117 admissible covering are considered. For each covering the Haar transform is applied to its tetrominoes, this generates four low-pass coefficients and twelve tetrolet coefficients. The tetrolet transform selects the optimal covering in each image block from the alphabet $\{1, \ldots, 117\}$. For the coefficients of the high frequency, the larger value of variance information measurement of multi-scale decomposition coefficients corresponds to sharper brightness changes. Therefore fusion rule is to select the larger value of variance information measurement of the two coefficients at each pixel. First we calculate the $12 \times 1$ tetrolet high frequency coefficients variance. Then the coefficient with larger variance is used in the following fusion process:

$$
\begin{gathered}
T_{F}(i, j)= \begin{cases}T_{A}(i, j) & \operatorname{Var}_{A}(i, j) \geq \operatorname{Var}_{B}(i, j) \\
T_{B}(i, j) & \operatorname{Var}_{B}(i, j) \geq \operatorname{Var}_{A}(i, j)\end{cases} \\
C_{F}(i, j)= \begin{cases}C_{A}(i, j) & \operatorname{Var}_{A}(i, j) \geq \operatorname{Var}_{B}(i, j) \\
C_{B}(i, j) & \operatorname{Var}_{B}(i, j) \geq \operatorname{Var}_{A}(i, j)\end{cases}
\end{gathered}
$$

where $(i, j)$ and $\operatorname{Var}(i, j)$ denotes the current position of tetrolet coefficient variance respectively. $C(i, j)$ denotes the current position of tetrolet coefficient covering number in each image block.

\section{Experiments and Results}

The proposed rules were used to implement the fusion of the multiband SAR image using complete tetrolet decomposition and the methods proposed above were tested for image fusion of the multi-band SAR image. Figure 2 (a) and (b) are two remote images with different band. Figure 2 (c) and (d) shows that fused image with the wavelet and proposed image fusion algorithms. 


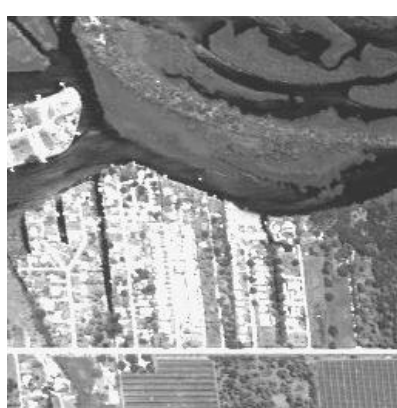

(a) original band 8 image

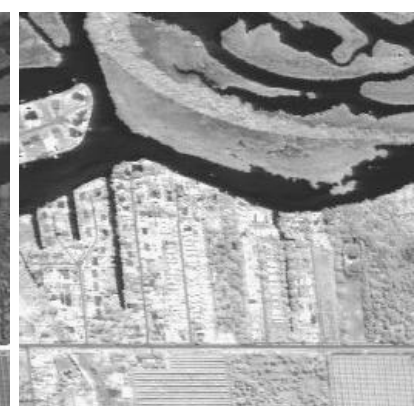

(b) original band 3 image

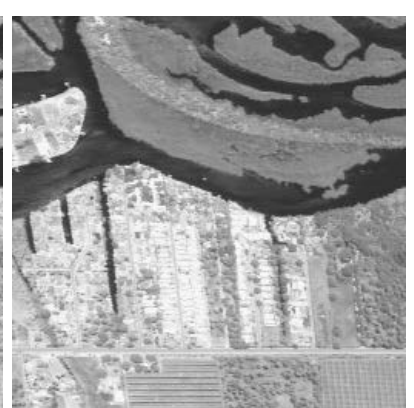

(c) fused image by WT

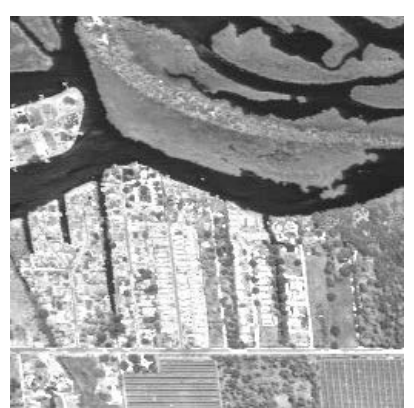

(d) proposed method

Figure 2: Fused image with the different methods. (a) The original band 8 image, (b) the original band 3 image, (c) fused image by WT, (d) fused image by proposed method

Some statistics factors were employed for evaluating the fusion effect such as entropy, cross entropy, mean and standard deviation ${ }^{[8]}$. The proposed fusion methods are compared with the wavelet transforms method. Criteria values of fused images by different methods are shown in Table 1

Table 1 Criteria value of fused images by different methods

\begin{tabular}{|c|c|c|c|c|c|}
\hline method Criteria & $\begin{array}{l}\text { average } \\
\text { value }\end{array}$ & $\begin{array}{l}\text { standard } \\
\text { deviation }\end{array}$ & entropy & $\begin{array}{c}\text { the mean } \\
\text { cross entropy }\end{array}$ & $\begin{array}{l}\text { the root } \\
\text { cross entropy }\end{array}$ \\
\hline WT & 150.801 & 53.934 & 7.4736 & 0.5844 & 0.6213 \\
\hline CT & 149.187 & 59.961 & 7.5054 & 0.4414 & 0.4517 \\
\hline
\end{tabular}

\section{Conclusion}

We explored the capabilities of the tetrolet transform for multi-band SAR images fusion. Tetrolet transform represents edges better than wavelet and it is well suited for the SAR image fusion. We designed variance information measurement for directional high-frequency coefficients in multiscale tetrole transform domain. Experiments and their studies were conducted by applying the proposed method, and also other image fusion methods. Both subjective and objective performance evaluation results can be drawn that this image fusion algorithm gives more satisfactory results than other methods

\section{Acknowledgment}

This work was financially supported by the Chinese National Science Foundation Grant (No.61272025 \& No.61402004) and the Nature Science Foundation of Anhui Province Education Department under Grant (No. KJ2012B065) and the Nature Science Foundation of Anhui Sanlian University Grant (No. 13ZLGC043 \& 2014Z020) and the revitalization plan project of higher education of Anhui Province Grant (No. 2013ZYTZ082).

\section{References}

[1] Wald, L., Ranchin, T. and Mangolini, M., Photogrammetric Engineering \& Remote Sensing. Vol. 63(1997), p. 691

[2] Myungjin Choi, Rae Young Kim,etal. IEEE Geoscience and Remote Sensing Letters, Vol.2 (2005), p. 136.

[3] Piella G. Information fusion, Vol. 4 (2003), p. 259.

[4] M. N. Do and M. Vetterli, IEEE Trans. on Image Processing, Vol.14 (2005), p. 2091.

[5] Zhang Jingjing, Fang Yonghua. Opto-Electronic Engineering, Vol. 34 (2007), p.78.

[6] S.W. Golomb. Polyominoes. Princeton University Press, 1994.. 
[7] Krommweh J. Journal of Visual Communication and Image Representation, Vol.21(2010),p.364.

[8] Zheng Yongan, Song Jianshe, Zhou Wenming. Systems Engineering and Electronics, Vol. 28 (2006), p.463. 